review is the direction it must help to give to future studies of the pathology of the root system.

It remains to discuss the form and purpose of this publication. The book is an undoubted sign of the increasing interest being taken in land cultivation and especially in its scientific foundations. It is a present need of all who cultivate the soil that they shall understand adequately what they are trying to do, from a biological as well as from an economic aspect, for the two are indissolubly related. It is for these people rather than for the biological specialist that this book is, presumably, intended. If so, then it is doubtful whether in its present form it adequately fulfils the needs of the public it is desired to reach. The manner of compilation has resulted in a great deal of duplication of matter, which is rather tiring to the reader and by no means makes for clarity of exposition. The style of writing is also often difficult ; this is partly because the papers were written for the instructed biologist with whom neither farmer, forester, nor horticulturalist is necessarily to be classed. No doubt the exigencies of the times made a re-writing of the work completely impossible; but it is to be hoped that at some future time the authors will be able to give a more connected and lucid account of the important relationships which exist between the root, the micro-flora of the soil and their physical environment. In the meantime, those who read through this series of papers will find much of interest and value. The many illustrations and the clear and well set out tables and text-figures are an undoubted help to their reading and understanding.

W. R. DAY.

\section{ELLIPTIC FUNCTIONS}

\section{Jacobian Elliptic Functions}

By Eric Harold Neville. Pp. xv+332. (Oxford : Clarendon Press ; London : Oxford University Press, 1944.) 25s. net.

$\mathrm{N}$ the preface to his "Elliptic Functions", written 1 in 1892, Prof. A. G. Greenhill complained that, although the subject was then nearly seventy years old, it had not made its way into the ordinary curriculum of mathematical study in Great Britain. Fifty years later, in a lecture to the London Mathematical Society, Prof. E. H. Neville declared that, while his contemporaries at Cambridge thirty-five years ago would have regarded the elements of the theory of Jacobian elliptic functions as a subject which every undergraduate should study, the time had now come when the subject was largely neglected. $\mathrm{He}$ claimed that the study of Jacobian elliptic functions was being killed by the unnatural way in which the functions were introduced, and that every general principle was stifled by the lack of symmetry and the multitude of special formulæ. The book under review is his attempt to restore the Jacobian functions to their proper place in a university curriculum.

An elliptic function is a doubly-periodic meromorphic function of a complex variable $z$. The Argand plane is divided into a lattice of periodparallelograms, and the elliptic functions are functions defined on this lattice. The simplest elliptic functions are of order two ; one type is the Weierstrass function $\mathfrak{p}(z)$, with a double pole in each cell; the other comprises the Jacobian functions with two simple poles per cell. While it is the case that $p(z)$ is defined directly on the lattice, the modern treatment of the Jacobian functions is not a direct one; the functions are introduced in an artificial way, undoubtedly the best way for the computer, by means of theta functions. In Prof. Neville's book, the Jacobian functions are defined directly by means of the Weierstrass function.

This is not entirely an innovation, but it is coupled with a new notation which exhibits the systematic and organic relations between the functions in a strikingly simple way. Writing $\omega_{f}, \omega_{g}, \omega_{h}$ for the halfperiods $\omega_{1}, \omega_{2}, \omega_{3}$ of Weierstrass, Prof. Neville introduces three primitive elliptic functions defined by

$$
\text { pj } z=\sqrt{ }\left\{p(z)-e_{p}\right\} \quad(p=f, g, h)
$$

with the condition that the residue at $O$ is unity; then pj $z$ has periods $4 \omega_{p}, 2 \omega_{q}, 2 \omega_{r}$, a simple pole at $O$ and a simple zero at $\omega_{p}$. To complete the notation, he uses a symbol $\omega_{j}$ to represent the origin, and denotes the function with a simple zero at $\omega_{p}$, $\mathbf{a}$ simple pole at $\omega_{q}$, by pq $z$. There are twelve functions in the complete set, and they are connected by the simple relations

$$
\begin{aligned}
& \text { pq } z \text { qr } z=\text { pq } \omega_{r} \text { pr } z \\
& \text { pq } z \text { qp } z=\text { pq }^{\prime} \omega_{q} \\
& \text { pj }^{2} z=\mathrm{qj}^{2} z+\mathrm{p}_{q}{ }^{2}
\end{aligned}
$$

where $\mathrm{p}_{q}$ is the value of $\mathrm{pj} \omega_{q}$.

The Jacobian function $u=\operatorname{sn} x$ was originally defined by the elliptic integral

$$
x=\int_{0}^{u} \frac{d u}{\sqrt{ }\left\{\left(1-u^{2}\right)\left(1-k^{2} u^{2}\right)\right\}} .
$$

To link up the new theory with the old, it is next shown that the relation

$$
z=\int_{0}^{w} \frac{d w}{\sqrt{ }\left\{\left(g^{2}-w^{2}\right)\left(g_{h}^{2}-w^{2}\right)\right\}}
$$

is equivalent to $w=-\mathrm{jg} z$. Comparing these integrals, we see that with $z=x$, the relations are identical if $g_{f}=1, g_{h}=-k, w=k u$. The actual dimensions and orientation of the lattice on which the primitive functions pj $z$ are defined is immaterial because of their homogeneity in $z$ and the three periods. The condition $g=1$ picks out of the whole family of similar lattices a special lattice called a Jacobian lattice. If the functions pq $z$ are defined on a Jacobian lattice and are suitably normalized, they are simply the twelve Jacobian elliptic functions ; in fact, $\mathrm{fj} z$, gj $z, \mathrm{hj} z$ are the functions $\operatorname{cs} u$, ns $u$ and $\mathrm{ds} u$ respectively.

Having now reached the customary notation, Prof. Neville again breaks with tradition. Instead of the usual quarter-periods $K, i K^{\prime}, K+i K^{\prime}$, he writes $K_{c}, K_{n}$ and $-K_{d}$, and uses $K_{s}$ as a symbol for the origin. This notation is likely to prove valuable, as it simplifies the whole presentation of the theory. For example, in the discussion of the trans. formations of Landen and Jacobi, the reader sees at once why the transformations work, as well as how.

It remains to complete the link with the definition of sn $u$ by the inversion of an elliptic integral. This part of the book is very well written. It emphasizes clearly the nature of the problem, and gives a solution on entirely new lines.

In this review it has been possible to mention only the salient points of an excellent book. We hope that it will have the success it deserves, and that it will revive interest in this fascinating branch of analysis.
E. T. Copson. 